\title{
Avaliação dos Distúrbios do Sono de pacientes submetidos à Polissonografia
}

\author{
Evaluation of the Sleep Disorders in patients submitted to Polissomnography \\ Evaluación de los Disturbios del Sueño de pacientes sometidos a la Polisomnografía
}

Recebido: 06/01/2021 | Revisado: 14/01/2021 | Aceito: 17/01/2021 | Publicado: 20/01/2021

Izabela dos Santos Barbosa

ORCID: https://orcid.org/0000-0001-8869-4411 Universidade Estadual de Mato Grosso do Sul, Brasil E-mail: izabela.sb@hotmail.com José Carlos Souza

ORCID: https://orcid.org/0000-0003-4460-3770 Universidade Estadual de Mato Grosso do Sul, Brasil E-mail: josecarlossouza@uol.com.br

Paulo de Tarso Guerrero Muller

ORCID: https://orcid.org/0000-0002-7724-245X

Universidade Federal de Mato Grosso do Sul, Brasil E-mail: paulo.muller@ufms.br

Paola Oliveira Cavalcante Brito

ORCID: https://orcid.org/0000-0002-6537-7816 Hospital Universitário Maria Aparecida Pedrossian, Brasil E-mail: paola.c@icloud.com

Caroline Torres Augusto

ORCID: https://orcid.org/0000-0003-4331-7414 Universidade Federal de Mato Grosso do Sul, Brasil E-mail: caroltaugusto@gmail.com

Isabella de Azevedo Cardeliquio Cantarelli ORCID: https://orcid.org/0000-0002-2889-3336 Universidade do Vale do Sapucaí, Brasil E-mail: isacantarelli@outlook.com

\begin{abstract}
Resumo
Objetivo: caracterizar os distúrbios do sono dos pacientes submetidos à polissonografia e identificar suas associações com variáveis epidemiológicas. Métodos: estudo transversal, observacional, conduzido com análise de dados dos prontuários de 101 pacientes submetidos a polissonografia no Laboratório de Medicina do Sono do HUMAP, Campo Grande, MS. Os dados utilizados foram os laudos da polissonografia e escalas padronizadas (escala de sonolência de Epworth e Questionário de Berlim). Resultados: O alto risco para Síndrome da Apneia Obstrutiva do Sono (SAOS) foi identificado em $83 \%$ dos avaliados. Quanto ao perfil polissonográfico, 77\% foram diagnosticados com SAOS, sendo a maioria representada pelo sexo feminino. Dos pacientes com SAOS, 95,5\% apresentava obesidade ou sobrepeso. A Sonolência Excessiva Diurna foi identificada em 51,2\% dos pacientes com diagnóstico de SAOS. Conclusão: A prevalência de distúrbios do sono foi elevada, sendo que os pacientes com diagnóstico de SAOS, maioria sexo feminino, apresentaram com maior frequência SED, sobrepeso ou obesidade e Hipertensão Arterial Sistêmica, fatores de risco importantes para a síndrome respiratória.
\end{abstract}

Palavras-chave: Polissonografia; Transtornos do sono-vigília; Síndrome da Apneia Obstrutiva do Sono.

\begin{abstract}
Objective: To characterize the sleeping disturbs of patients submitted to a polysomnography and establish their associations with epidemiological ranges. Methods: Transversal study, observational, conducted by analyzing data of 101 patients submitted to polysomnography at the Laboratory of Sleep Medicine of HUMAP, Campo Grande, MS, Brazil. The data used were the polysomnography itself and patronized grades (somnolence scale of Epworth and Berlin Questionnaire). Results: The high risk for Syndrome of Obstructive Sleep Apnea (SOSA) was identified in $83 \%$ of the evaluated patients. About the polysomnographic results, $77 \%$ were diagnosed with SOSA, being most of them female. In the patients with SOSA, 95,5\% were obese or overweighed. The excessive daytime sleepiness was detected in $51,2 \%$ of the patients diagnosed with SOSA. Conclusion: The prevalence of sleeping disorders was high, being the patients that were diagnosed with SOSA most of them female, with a bigger SED frequency, overweighed and obese and with high blood pressure. These were relevant risk factors for a respiratory syndrome.
\end{abstract}

Keywords: Polysomnography; Sleep wake disorders; Syndrome of Obstructive Sleep Apnea. 


\begin{abstract}
Resumen
Objetivo: Caracterizar los disturbios del sueño de los pacientes sometidos a la polisomnografía e identificar sus asociaciones con variables epidemiológicas. Métodos: Estudio transversal, observacional, conducido con análisis de datos de los prontuarios de 101 pacientes sometidos a polisomnografía en el Laboratorios de Medicina del Sueño HUMAP, Campo Grande, MS. Los datos utilizados fueron los laudos de la polisomnografía y escalas estandarizadas (escala de somnolencias de Epworth y cuestionario de Berlín). Resultados: El alto riesgo para El Síndrome de la Apnea Obstructiva del Sueño (SAOS) fue identificado en $83 \%$ de los evaluados. En cuanto al perfil polisomnográfico, $77 \%$ fueron diagnosticados con SAOS, siendo la mayoría representada por el sexo femenino. De los pacientes con SASOS, 95,5\% presentaba obesidad o sobrepeso. La Somnolencia excesiva Diurna fue identificada en un 51,2\% de los pacientes con diagnóstico de SAOS. Conclusión: La prevalencia de disturbios del sueño fue elevada, siendo que los pacientes con diagnóstico de SAOS, mayoría sexo femenino, presentaban con mayor frecuencia SED, sobrepeso u obesidad e Hipertensión Arterial Sistémica, factores de riesgo importantes para el síndrome respiratorio.
\end{abstract}

Palabras clave: Polisomnografía; Trastornos del sueño-vigilia; Síndrome de Apnea Obstructiva del Sueño.

\title{
1. Introdução
}

O sono é uma condição fisiológica e vital regulado por redes neurais complexas que desempenham um papel importante na regulação do sistema nervoso e nas funções biológicas do corpo (Santos et al., 2014, Morais, 2012). Processo ativo caracterizado por modificação do estado de consciência, essencial para equilíbrio homeostático, restauração metabólica, consolidação da memória, entre outras (Morais, 2012, Ohayon et al., 2018). A alternância entre sono e vigília ocorre de forma circadiana, sendo este ciclo variável de acordo com fatores endógenos, sociais e ambientais (Neves et al., 2017, Pereira, 2013).

O sono é composto por dois estados distintos: rapid eye movement (REM) e non-rapid eye movement (NREM), os quais manifestam-se em ciclos organizados ao longo de uma noite. O sono REM ocupa aproximadamente 20 a $25 \%$ do tempo de sono de adultos jovens, variando de 5 a 30 minutos. Esta fase caracteriza-se pela presença de sonhos e movimentos corporais. Enquanto o sono NREM, que ocupa cerca de 75\% do ciclo do sono, é divido em três estágios (N1, N2 e N3), sendo o último estágio o mais profundo (Santos et al., 2014, Manzoli et al., 2018).

As alterações do padrão de sono são comuns em todas as faixas etárias, mas deve-se destacar que estudos internacionais indicam alta prevalência de distúrbios do sono em adultos (Zanuto et al., 2015). A privação do sono, condição observada cada vez mais na sociedade moderna, pode levar ao aumento da sonolência diurna, fadiga, diminuição do desempenho ao executar atividades, diminuição da capacidade de memorização, prejudicando a produtividade global e a saúde (Ribeiro et al., 2013).

Estudos recentes identificaram a associação entre distúrbios do sono e o aumento da morbidade e mortalidade. Isso deve-se aos efeitos sistêmicos exercidos, com alterações nas vias endócrina, metabólica e imunológicas, relacionadas a desfechos de saúde desfavoráveis, como depressão, diabetes mellitus, hipertensão arterial sistêmica, obesidade, as quais também contribuem para o desenvolvimento de doenças cardiovasculares (Jeddi et al., 2016, Galli et al. 2013).

Considerando os transtornos do sono, a Classificação Internacional dos Transtornos do Sono (ICSD) os agrupa de acordo com os sintomas e categorias que relacionem entre si (Neves et al., 2017). De acordo com a ICSD-3, os transtornos do sono podem ser classificados nos seguintes grupos: Insônia; Distúrbios respiratórios relacionados ao sono; Distúrbios centrais de hipersonolência; Distúrbios do ritmo circadiano relacionado ao ciclo sono-vigília; Parassonias; Distúrbios do movimento relacionados ao sono e outros distúrbios do sono (Santos et al., 2014, Gonçalves, 2016, Sateia, 2014, American Academy of Sleep Medicine, 2014).

A avaliação de um paciente baseia-se na observação clínica, com enfoque na história e exame físico. Já a avaliação subjetiva inclui aplicação de questionários e diários de sono. Enquanto a avaliação objetiva é realizada por meio de exames complementares variados, sendo o principal, a polissonografia (PSG) (Santos et al., 2014, Morrison \& Riha, 2012). O exame é realizado em laboratório e constitui-se no método padrão ouro para diagnóstico dos transtornos do sono, principalmente os 
respiratórios. Consiste na monitorização contínua da atividade encefálica, movimento dos olhos, respiração e movimento dos membros inferiores, e tem sido usada há décadas (Santos et al., 2014, Morrison \& Riha, 2012, Deepak Shrivastava, 2014).

Ainda que seja elevada a prevalência de distúrbios do sono na população mundial, e esse desfecho esteja relacionado à maior ocorrência de complicações e despesas com saúde, os dados e estudos epidemiológicos nacionais, em especial, estaduais, são incipientes. O objetivo do presente estudo foi caracterizar os distúrbios do sono de pacientes atendidos no Laboratório do Sono de um serviço público de Campo Grande, Mato Grosso do Sul, bem como identificar suas associações com variáveis epidemiológicas.

\section{Metodologia}

A pesquisa constituiu em um estudo transversal, descritivo, observacional, de natureza quantitativa (Pereira et al., 2018). O Hospital Universitário Maria Aparecida Pedrossian (HUMAP), localizado em Campo Grande, MS conta com um Laboratório do Sono, coordenado pelo Serviço de Pneumologia e Medicina Respiratória da instituição. Dessa forma, a coleta dos dados dos prontuários de pacientes submetidos à polissonografia ocorreu entre fevereiro e junho de 2019

Os dados foram coletados à partir de 101 prontuários físicos dos pacientes submetidos a polissonografia no período de 2015 a 2019. Como instrumento de coleta de dados foi utilizado um formulário, criado pelos próprios pesquisadores com objetivo de padronizar as informações coletadas.

Em relação às variáveis, foram avaliadas: sexo, idade, estado civil, hábitos de vida e de sono, comorbidades, medicações em uso, Sonolência excessiva diurna (SED) pelo questionário de Epworth e risco para Síndrome da Apneia Obstrutiva do Sono (SAOS), pelo questionário de Berlim; e dados da polissonografia. Importante destacar que o estudo foi aprovado pelo Comitê de Ética em Pesquisa da Universidade Federal de Mato Grosso do Sul, protocolo número 3.207.250, seguindo os preceitos éticos.

A comparação entre as variáveis contínuas foi realizada pelo teste $t$ de Student. A comparação entre as variáveis qualitativas foi realizada pelo teste qui quadrado. Esse processo levou em conta intervalos de confiança de 95\% (IC 95\%), valores de $\mathrm{p}<0,05$ foram considerados estatisticamente significantes. Os pacotes de software Microsoft Office Excel e BioEstat (versão 5.0) foram utilizados para construir e analisar os dados.

\section{Resultados}

Dos 101 prontuários, obteve-se que a média de idade dos avaliados foi de 51,2 anos ( $\pm 14,3$ anos (IC: $95 \%)$ ) e os indivíduos foram subdivididos em faixas etárias, sendo observado predomínio de adultos (67\%). Em relação ao sexo, 56\% dos avaliados eram do sexo feminino e $44 \%$, masculino. A análise do peso mostrou que $87 \%$ dos pacientes avaliados, de acordo com o Índice de Massa Corpórea (IMC), apresentavam sobrepeso ou obesidade, ou seja, IMC superior a 24,9km/m2.

No que tange aos hábitos de vida $8 \%$ dos avaliados declararam ser tabagistas e $36 \%$, consumir bebida alcoólica. Quanto ao uso de medicamentos para dormir $21 \%$ referiram fazer uso de alguma classe de hipnótico. Já em relação ao consumo de cafeína $62 \%$ declararam ingerir até 2 xícaras ou copos por dia.

Quanto aos hábitos de sono a maioria dorme acompanhada, algumas vezes apresenta problemas para pegar no sono e mantê-lo, acordar para ir ao banheiro e se manterem acordados. De modo geral, os sintomas noturnos foram relatados por poucos pacientes, resultando em valores não representativos, e são eles: movimentos involuntários e repetitivos das pernas, câimbras, perda de força, alucinações, paralisia do corpo, pesadelos, terror, agitação, sonambulismo e bruxismo.

Em relação às comorbidades, a maioria dos pacientes (54,7\%) apresentava diagnóstico de Hipertensão Arterial Sistêmica (HAS), sendo que a análise individualizada para cada sexo mostrou prevalência relativa em $61 \%$ das mulheres e 
$29 \%$ homens. Na sequência, distúrbios respiratórios foram relatados por 28,8\% dos pacientes, diabetes $22 \%$, distúrbios cardíacos $21,1 \%$, neurológicos $11 \%$ e outros em menor proporção.

A sonolência excessiva diurna (SED), avaliada pela Escala de Epworth foi identificada em 53\% (H: 40\%; M: 60\%) dos pacientes que realizaram o exame. Já o alto risco para SAOS, avaliado pelo Questionário de Berlim, foi identificado em $83 \%$ (H:44\% M:56\%) dos pacientes que realizaram o exame.

Quanto à análise da polissonografia, 77\% dos pacientes foram diagnosticados com SAOS, sendo $15 \%$ leve, $22 \%$ moderada e $39 \%$ grave, $1 \%$ não obteve resultado conclusivo. Correlacionando as variáveis, em relação ao IMC, 95,5\% dos pacientes com diagnóstico de SAOS apresentavam obesidade ou sobrepeso e a SED foi identificada em 51,2\% dos pacientes com diagnóstico de SAOS.

\section{Discussão}

Os transtornos do sono estão entre os distúrbios clínicos com maior impacto na saúde. Estão relacionados ao ganho de peso, e a redução da qualidade do sono parece estar associada à maior ingestão alimentar e ao etilismo (Zanuto et al., 2015). A ocorrência de distúrbios relacionados ao sono foi elevada, considerando-se a ocorrência de SAOS e SED. Este percentual foi superior ao observado em estudos abordando o mesmo tema em populações de outros países e até mesmo no Brasil.

A obesidade está muito relacionada ao desenvolvimento e agravamento de distúrbios do sono (Luyster et al., 2012). Dessa forma, a elevada ocorrência encontrada pode estar associada ao excesso de peso (sobrepeso e obesidade) identificado em $87 \%$ dos pacientes. Neste grupo, a qualidade do sono pode ser afetada de diferentes formas. O excesso de tecido adiposo na região cervical interfere na circulação do ar e, por consequência, na respiração, podendo levar a SAOS, por exemplo (Silva et al., 2014, Pissulin et al., 2018).

Correlacionando as variáveis, em relação ao IMC, 95,5\% dos pacientes com diagnóstico de SAOS apresentavam obesidade ou sobrepeso. Um estudo realizado no Instituto do Sono de Santa Maria (ISSM) avaliou a polissonografia basal de noite inteira de 88 pacientes com diagnóstico de SAOS. Concluiu que a obesidade estava associada a alterações polissonográficas que expressam baixa qualidade do sono em pacientes obesos (Piccin et al. 2015).

Em relação aos hábitos de vida sabe-se que o alcoolismo interfere negativamente na qualidade do sono, já que altera o ritmo circadiano, a sequência e duração dos ciclos do sono, com despertares noturnos, diminuindo sua eficiência e aumentando a sonolência diurna (Talhada, 2012). Neste estudo, a maioria dos pacientes (64\%) avaliados referiram não consumir bebida alcoólica.

Quanto ao tabagismo, os efeitos nocivos do tabaco já são bem elucidados, no entanto, a relação do tabagismo com a qualidade do sono não está completamente estabelecida (Talhada, 2012). Em relação aos problemas respiratórios, sabe-se que os fumantes tem maior risco para apneia obstrutiva do sono (Moreno et al., 2018). No presente estudo 66\% dos tabagistas apresentaram diagnóstico de SAOS. No estudo de Kim et al. foi observado que o tabagismo pode desencadear distúrbios do sono e até ser um fator de risco para apneia obstrutiva do sono (Kim et al., 2017).

No que tange às comorbidades, 54,7\% referiram diagnóstico de HAS, sendo a maioria representada pelo sexo feminino. Deve-se destacar que a SAOS se correlaciona com os seguintes fatores: HAS, obesidade, dislipidemias e diabetes mellitus - com importante interrelação entre eles (Kanda et al., 2018). Outra alteração relacionada à SAOS é a Sonolência Excessiva Diurna, identificada em 53\% (H: 40\%; M: 60\%) dos pacientes. Definida como a propensão aumentada ao sono,resultando em sonolência e lapsos de sono não intencionais; pode ocorrer por privação de sono crônica, uso de medicamentos, narcolepsia, hipersonia idiopática e SAOS (Matnei et al., 2017).

A análise percentual do risco para SAOS através do Questionário de Berlim, revelou que 83\% (H:44\% M:56\%) dos 
pacientes que se submeteram ao exame apresentaram alto risco para SAOS. Já em relação as impressões diagnósticas pode-se observar que 20,6\% dos pacientes obtiveram padrão dentro da normalidade, 15,5\% SAOS leve, 22,4\% SAOS moderada, $39,6 \%$ SAOS grave e apenas 1,7\% não obteve diagnóstico conclusivo.

Diferente do que foi encontrado na literatura, a frequência de SAOS foi maior no sexo feminino (58\%) em relação ao masculino. De forma geral, mulheres apresentaram maior ocorrência de distúrbios do sono. Biologicamente, as mulheres possuem um sono mais fragmentado, tornando-o menos contínuo quando comparado ao dos homens (Zanuto et al., 2015, Kanda et al., 2018). Além disso, o estressante contexto social no qual as mulheres estão inseridas (cobrança social referente ao trabalho, cuidados com a família, estética entre outros) pode fazer com que elas assumam comportamentos não saudáveis e que impactam negativamente no sono (Zanuto et al., 2015, Matnei et al., 2017).

Por fim, quanto aos os sinais observados durante o exame, houve predomínio de ronco. Já o bruxismo, arritmias e microdespertares foram pouco identificados durante o exame. $\mathrm{O}$ ronco, assim como a presença de apneia, pode ser exacerbado após a ingestão de álcool ou do aumento de peso (Matnei et al., 2017). Como identificado nesta pesquisa, o ronco foi relatado em todos os pacientes com diagnóstico de SAOS.

Pode-se concluir deste trabalho que dentre os pacientes que realizaram o exame de polissonografia houve alto risco para SAOS, avaliada pelo questionário de Berlim e considerável presença de Sonolência Excessiva Diurna, avaliada pelo questionário de Epworth. A SAOS, identificada na maioria dos avaliados esteve relacionada ao sexo feminino, HAS, peso e IMC elevados.

É importante destacar a necessidade de cautela na extrapolação dos dados para a população geral, tendo em vista que os pacientes avaliados podem não refletir a realidade da população brasileira geral. Ainda assim, os resultados encontrados podem contribuir para que sejam traçadas estratégias de intervenção no sentido de prevenir ou atenuar a ocorrência de distúrbios do sono eliminando os fatores de risco modificáveis relacionados a eles. A partir dos dados já coletados pretende-se ampliar a amostra e produzir conteúdo científico com temáticas centrais direcionadas, contribuindo assim, para ampliação dos dados epidemiológicos.

\section{Considerações Finais}

O sono possui papel importante na saúde e bem-estar, na atividade cognitiva, memória e no processo de aprendizagem dos indivíduos. Por isso é fundamental que este ocorra de modo satisfatório, visando favorecer o bom desempenho da pessoa em todos os espaços que possa estar inserida.

Já é de conhecimento geral que o sono tem um papel vital, fundamental para consolidação restauração da energia, do metabolismo. Diante das suas importantes funções, os distúrbios do sono podem implicar alterações significativas no funcionamento biológico, cognitivo, social, refletindo diretamente na qualidade de vida do indivíduo.

A pesquisa, pioneira no Estado de Mato Grosso do Sul, permitiu que houvesse uma quantificação e identificação dos principais distúrbios do sono. Além disso, conforme proposto inicialmente, foi possível identificar o perfil sociodemog ráfico e epidemiológico da população estudada, bem como os hábitos de vida e de sono.

Considerando os distúrbios do sono identificados, a maioria dos pacientes foi diagnosticada com SAOS, condição que interfere significativamente na qualidade do sono, qualidade de vida aumentando o risco para o desenvolvimento de outras patologias. A condição identificada desperta atenção para a importância do cuidado com o sono como um todo, muitas vezes negligenciado pela população em geral. Em relação à obesidade, condição identificada na maioria dos pacientes, para além dos distúrbios do sono, é considerada fator de risco para inúmeras patologias que comprometem a qualidade de vida e bem estar.

O trabalho desenvolvido no Laboratório do Sono permitiu a elucidação do perfil dos pacientes atendidos no serviço, 
bem como suas necessidades. Dessa forma, torna-se fundamental um novo olhar, dos profissionais da saúde do Estado, com intuito de planejar e instituir medidas de prevenção e promoção da saúde com enfoque na medicina do sono.

Apesar da necessidade de cautela na extrapolação dos dados para a população geral, os resultados contribuíram para o enriquecimento dos dados epidemiológicos do Estado. Além disso, a pesquisa realizada em uma instituição pública, despertou o interesse ampliar os dados e realizar novos estudos abrangendo uma maior amostra, garantindo maior representatividade dos dados.

\section{Agradecimentos}

O agradecimento é direcionado ao Conselho Nacional de Desenvolvimento Científico e Tecnológico (CNPq) pela concessão da bolsa para o desenvolvimento de projeto de Iniciação Científica pela Universidade Estadual de Mato Grosso do Sul.

\section{Referências}

American Academy of Sleep Medicine. (2014). International Classification of Sleep Disroders, 3ed. Diagnostic and coding manual. American Academy of Sleep Medicine.

Deepak Shrivastava, M. D., Syung Jung, M. D., Mohsen Saadat, D. O., Roopa Sirohi, M. D., \& Keri Crewson, M. D. (2014). How to interpret the results of a sleep study. Journal of Community Hospital Internal Medicine Perspecties, 4(5), 1-4.

Galli, G., Piaggi, P., Mattingly, M. S., Jonge, L., Courville, A. B., Pinchera, A., Santini, F., Csako, G., \& Cizza, G. (2013). Inverse relationship of food and alcohol intake to sleep measures in obesity. Nutrition \& Diabetes, 3, e58.

Gonçalves, D. A. R. (2016). Descrição de parâmetros polissonográficos relacionados à insônia e à fragmentação do sono em pacientes com diagnósticos de condições otorrinolaringológicas que causam obstrução nasal. [Dissertação de Mestrado em Ciências Médicas, Faculdade de Medicina da Universidade de Brasília, Brasília].

Jeddi, S., Asl, A. N., Asgari, A., \& Asghar, G. (2016). O efeito da privação de sono na função cardíaca e tolerância à lesão de isquemia-reperfusão em ratos. Arquivos Brasileiros de Cardiologia, 106(1), 41-48.

Kanda, G. M., Priore, N. C., Toledo, C., Shimizu, R. N., Arata, Y. P., Gonzaga, C., Bertolami, A., Amodeo, C., \& Minuzzo, L. (2018). Perfil clínico e laboratorial de pacientes com e sem apneia obstrutiva do sono. Revista da Sociedade Brasileira de Clínica Médica, 16(2), 108-112.

Kim, S. Y., Sim, S., \& Choi, H. G. (2017). High stress, lack of sleep, low school performance, and suicide attempts are associated with high energy drink intake in adolescents. PLoS One, 12(11): e0187759.

Kubota, A. M. A. (2013). Aspectos da insônia no adulto e a relação com o desempenho ocupacional. [Trabalho de Conclusão de Curso, Curso de Graduação em Terapia Ocupacional, Universidade de Brasília, Brasília].

Luyster, F. S., Strollo, P. J. J., Zee, P. C., \& Walsh, J. K. (2012). Sleep: A health imperative. Sleep; 35(6), $727-734$.

Manzoli, J. P. B, Correia, M. D. L, \& Duran, E. C. M. (2018). Conceptual and operational definitions of the defining characteristics of the nursing diagnosis Disturbed Sleep Pattern. Revista Latino-Americana de Enfermagem, 26, e3105. http://dx.doi.org/10.1590/1518-8345.2582.3105

Matnei T., Deschk, M. A. S., Sabatini, J. S., Souza, L. P., Santos, R. F., \& Camargo, C. H. F. (2017). Correlação da Escala de Sonolência de Epworth com alterações na polissonografia na avaliação da sonolência excessiva diurna em pacientes com síndrome da apneia-hipopneia obstrutiva do sono. Medicina (Ribeirão Preto), 50(2), 102-108.

Morais, R. S. (2012). Prevalência de insônia em gestantes. [Trabalho de Conclusão de Curso, Curso de Graduação em Fisioterapia, Universidade Estadual da Paraíba, Campina Grande].

Moreno, C. R. C., Santos, J. L. F., Lebrão, M. L., Ulhôa, M. A., \& Duarte, Y. A. O. (2018). Sleep disturbances in older adults are associated to female sex, pain and urinary incontinence. Revista Brasileira de Epidemiologia; 21(Suppl 2), E180018.

Morrison, I., \& Riha, R. L. (2012). Excessive daytime sleepiness and narcolepsy--an approach to investigation and management. European journal of internal medicine, 23(2), 110-117.

Neves, G. S. M., Macedo, P., \& Gomes, M. M. (2017). Sleep Disorders: Up to date (1/2). Revista Brasileira de Neurologia, 53(3), 19-30.

Ohayon, M. M., Chen, M. C., Bixler, E., Dauvilliers, Y., Gozal, D., Plazzi, G., Vitiello, M. V., Paskow, M., Roach, A., \& Hirshkowitz, M. (2018). A provisional tool for the measurement of sleep satisfaction. Sleep Health 4, 6-12. https://doi.org/10.1016/j.sleh.2017.11.002

Pereira, A. R. S. (2013). Hábitos de sono em estudantes universitários. [Dissertação de Mestrado em Ciências Farmacêuticas, Universidade Fernando Pessoa, Faculdade de Ciências da Saúde, Porto].

Pereira A.S., Shitsuka, D. M., Parreira, F. J., Shitsuka, R. (2018). Metodologia da pesquisa científica. Santa Maria, RS: Ed. UAB/NTE/UFSM. 
Research, Society and Development, v. 10, n. 1, e40110111738, 2021

(CC BY 4.0) | ISSN 2525-3409 | DOI: http://dx.doi.org/10.33448/rsd-v10i1.11738

Piccin, C. F., Beck, M. C., Oliveira, L. C. A., Cóser Neto, R. F., Cóser, P. L., Scapini, F., \& Silva, A. M. V. (2015). Obesidade e variáveis polissonográficas em pacientes com apneia obstrutiva do sono. O Mundo da Saúde, 39(3), 299-306.

Pissulin, F. D. M., Pacagnelli, F. L., Alda, M. A., Beneti, R., Barros, J. L., Minamoto, S. T., Weber, S. A. T. (2018). Tríade síndrome da apneia obstrutiva do sono, DPOC e obesidade: Sensibilidade de escalas de sono e de questionários Respiratórios. Jornal Brasileiro de Pneumologia, 44(3), 202-206.

Ribeiro, C. R. F., Silva, Y. M. G. P., \& Oliveira, S. M. C. (2013). A qualidade ruim do sono de acadêmicos de medicina. Anais eletrônicos do $12^{\circ}$ Congresso Brasileiro de Medicina da Família e Comunidade. Belém.

Santos, L. C, Castro, N. J., Ruback, O. R., Trigo, T. J. B., \& Rocha, P. M. B. (2014). Transtornos do ciclo sono-vigília / Circadiano - uma revisão de literatura. Brazilian Journal of Surgery and Clinical Research, 7(2), 38-43.

Sateia, M. M. D. (2014). International Classifi cation of Sleep Disorders-Third Edition. CHEST; 146(5), 1387-1394.

Silva, H. G. V., Moreira, A. S. B., Santos, V. R., Santos, S. O., \& Rêgo, A. F. B. (2014). Factors associated with obstructive sleep apnea severity: Obesity and excessive daytime sleepiness. Revista Brasileira de Cardioogia, 27(2),76-82.

Talhada, L. C. R. M. (2012). Qualidade do sono, saúde e estilos de vida. Estudo com a população activa portuguesa. [Dissertação de Mestrado em Psicologia Clínica e da Saúde, Universidade da Beira Interior, Covilhã].

Zanuto, E. A. C., Lima, M. C. S., Araújo, R. G., Silva, E. P., Anzolin, C. C., Araujo, M. Y. C., Codogno, J. S., Christofaro, D. G. D., \& Fernandes, R. A. (2015). Distúrbios do sono em adultos de uma cidade do Estado de São Paulo. Revista Brasileira de Epidemiologia, 18(1), 42-53. http://dx.doi.org/10.1590/1980-5497201500010004 